\title{
Piomiositis en un paciente inmunocompetente
}

\author{
Ezequiel Baran, Karina Aguilera, Luciana M. Lorenzi, Raúl Simoneto, Laura Valuntas y Gabriel Basso
}

\section{Pyomyositis in an immunocompetent patient}

We report a case of pyomyositis in a 59 year-old immunocompetent male patient caused by methicillin-susceptible Staphylococcus aureus. A CT scan of the chest demonstrated a lesion of the pectoral muscles. The patient was treated with surgical drainage and cephalexin. Pyomyositis is a rare and potentially serious infection that requires early recognition and prompt treatment.

Key words: Pyomyositis, Staphylococcus aureus, infection.

Palabras clave: Piomiositis, Staphylococcus aureus, infección.

\section{Introducción}

L a piomiositis es una infección infrecuente y potencialmente grave, que afecta los músculos esqueléticos y no es secundaria a una infección contigua de la piel, huesos o tejidos blandos ${ }^{1,2}$. Se refiere a una colección localizada en el músculo, entidad diferente de la miositis o la mialgia difusa ${ }^{3}$.

Originariamente fue descrita en regiones tropicales por lo que se conoció inicialmente como piomiositis tropical; sin embargo, su incidencia en climas templados se encuentra en incremento ${ }^{4}$, con posterioridad al surgimiento de la epidemia del virus de inmunodeficiencia humana $(\mathrm{VIH})^{5} \mathrm{y}$ ha pasado a ser denominada en este medio como piomiositis no tropical.

Los grupos musculares que se afectan mayoritariamente se sitúan en la región proximal de los miembros inferiores y cintura pélvica. Los sitios menos afectados son la pared abdominal, torácica y paraespinales ${ }^{6,7}$. No obstante, cualquier músculo puede involucrarse y la distribución multifocal es la regla ${ }^{5}$.

Staphylococcus aureus es el patógeno causal más frecuente ${ }^{8}$.

Los factores de riesgo son diabetes mellitus, infección por VIH, enfermedades malignas, consumo de alcohol, colagenopatías y uso de corticosteroides ${ }^{8,9}$. Un tercio de los pacientes no presentan factores de riesgo ${ }^{10}$.

El reconocimiento temprano como el tratamiento oportuno son necesarios dado que rápidamente puede desarrollarse un cuadro grave ${ }^{3}$, y sus manifestaciones clínicas son similares a una celulitis o un absceso en las primeras etapas ${ }^{11}$.

\section{Caso clínico}

Varón de 59 años, nacido en Buenos Aires, vendedor de un comercio céntrico, no había efectuado viajes fuera de la ciudad en los últimos cuatro años, no poseía mascotas, realizaba una alimentación balanceada, con cuatro comidas diarias. No presentaba antecedentes patológicos de significación. Consultó en el Sanatorio IPENSA de La Plata por omalgia derecha al realizar abducción, aducción y rotación interna, con una data de una semana. Posteriormente presentó una placa eritematosa en la región torácica anterior de cuatro cm de diámetro, no indurada, sin signos inflamatorios superficiales. No presentaba fiebre, pérdida de peso, hemoptisis, ni historia previa de traumatismo.

Se le realizó una ecografía de tejidos blandos con la que se visualizó en la región anterior del hemitórax derecho una imagen heterogénea de 3,89 cm, ubicada a 2,46 cm bajo la piel, compatible con una celulitis. No presentaba lesiones en el miembro superior ipsilateral, adenopatías, soplo cardíaco ni dolores óseos.

Comenzó con tratamiento antimicrobiano (cefalexina 1 gramo cada $12 \mathrm{hs);} \mathrm{a} \mathrm{las} 48$ horas presentó un registro febril de $38,6^{\circ} \mathrm{C}$ y una tumoración en la región pectoral derecha.

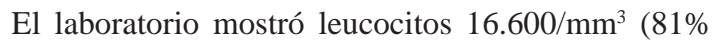
neutrófilos segmentados), hematocrito $40 \%$, hemoglobina 12,3 g\%, (VCM 89,7 fl), 250.000 plaquetas $/ \mathrm{mm}^{3}$, glicemia $85 \mathrm{mg} / \mathrm{dL}$, VHS $110 \mathrm{~mm}$ en la primer hora, TGO $40 \mathrm{U} / \mathrm{L}$ y TGP 70 U/L. La función renal y la creatin-fosfoquinasa (CPK) fueron normales. En la radiografía de tórax se observó ensanchamiento mediastinal (Figura 1), por lo que se solicitó una TAC de tórax y cuello con medio de contraste ev que demostró en la pared anterior del hemitórax derecho una lesión con densidad de tejidos blandos, que provocaba un aumento del espesor de los músculos pectorales. Dicha lesión se extendía hacia el mediastino anterior y superior, en proximidad con los grandes vasos y se asociaba a cambios densitométricos de la grasa mediastinal.
Sanatorio IPENSA, Buenos Aires, Argentina.

Servicio de Clínica Médica (EB). Servicio de Neumonología (EB) . Servicio de Infectología (KA). Servicio de Diagnóstico por Imágenes (LML, RS, LV). Servicio de Cirugía Torácica (GB).

Conflictos de interés: ninguno Fuente de financiamiento: ninguno

Recibido: 23 de marzo de 2011 Aceptado: 4 de enero de 2012

Correspondencia a:

Ezequiel Baran ezebaran@gmail.com 
Figura 1. Teleradiografía de tórax.

Figura 2. Punción aspirativa parietal y mediastinal guiada por tomografía axial computada.
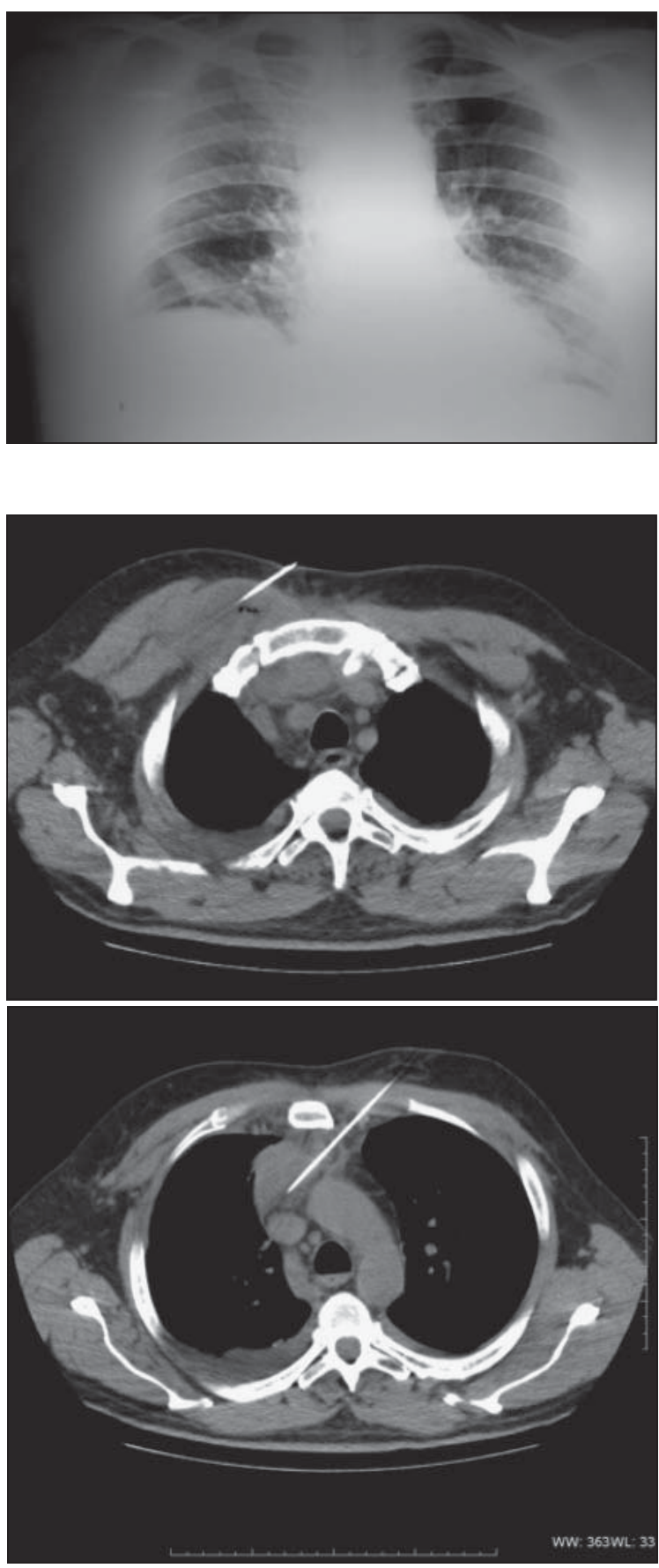

Se realizó una punción-aspirativa guiada por TAC (Figura 2), obteniéndose $25 \mathrm{ml}$ de material purulento de la colección parietal y $7 \mathrm{ml}$ de la mediastinal Se remitieron a estudio microbiológico y se ajustó el esquema anti-infeccioso a vancomicina 1 g cada 8 horas (dosis máxima), clindamicina 600 mg cada 6 horas y ceftazidima 2 g cada 8 horas ev.

Posteriormente se drenó quirúrgicamente el absceso pectoral. En la tinción de Gram se observaron cocáceas grampositivas agrupadas en racimos y al tercer día de tratamiento se informó la presencia de $S$. aureus sensible a oxacilina, cefalotina, eritromicina, clindamicina, cotrimoxazol, vancomicina y ciprofloxacina. Las tinciones de Ziehl-Neelsen, Giemsa y Gomori-Grocott resultaron negativas. Se modificó el tratamiento antimicrobiano indicado a cefazolina 2 g cada 8 horas ev. Las serologías para el virus de la inmunodeficiencia humana (VIH) y VDRL fueron no reactivas. El urocultivo, hemocultivos, cultivos para hongos y micobacterias del material purulento resultaron negativos. Un ecocardiograma bidimensional transtorácico no evidenció vegetaciones valvulares. La ecografía abdominal no presentó alteraciones. Al cabo de 72 hs se encontraba afebril, con mejoría clínica evidente. Dada la buena respuesta postquirúrgica y habiendo aislado en todas las muestras $S$. aureus sensible a meticilina se cambió tratamiento a cefalexina 1 g cada 8 horas vía oral, evolucionando favorablemente.

\section{Discusión}

La piomiositis es la infección supurativa del músculo estriado. Ocurre predominantemente en zonas tropicales, condición habitualmente observada en niños. En la piomiositis no tropical, dos tercios de los casos son adultos y sólo 9\% de los pacientes reportados presentan viajes o son inmigrantes de zonas tropicales ${ }^{10}$. La infrecuencia de la enfermedad se debe a la resistencia de los músculos a la infección.

La patogénesis se desconoce con exactitud. Se ha propuesto que receptores fijadores de fibronectina en las células musculares sirven como vía de entrada bacteriana, y es necesario el trauma muscular previo para su desarrollo. Se ha sugerido el ejercicio vigoroso y el trauma local como factores de riesgo, pero tan solo un tercio de los pacientes lo evidencian. En pacientes infectados con VIH la causa de daño muscular puede incluir toxicidad mitocondrial provocada por medicación anti-retroviral (por ejemplo: zidovudina). Los pacientes con otro tipo de inmunocompromiso e infectados con VIH son particularmente susceptibles a piomiositis bacteriana debido a la disfunción de los neutrófilos, especialmente al defecto del metabolismo oxidativo que predispone a infecciones por microorganismos catalasa-positiva como es S. aureus ${ }^{4}$.

Los síntomas clínicos presentan tres estadios. El primero se caracteriza por molestia muscular, fiebre baja y decaimiento. Dado que sólo la aponeurosis se encuentra inervada, el dolor sobre el músculo demora en aparecer, entre una y dos semanas, luego se produce la conformación del absceso. Durante el segundo estadio, la conformación del absceso comienza a ser sintomática con dolor, rigidez muscular y sepsis. A la mayoría de los 
pacientes se los reconoce en este momento evolutivo. De no ser tratado, la enfermedad evoluciona a un tercer estadio, con destrucción muscular, extensión local con osteomielitis, u osteoartrosis, septicemia y diseminación a distancia ${ }^{12}$. Se han descrito casos de síndrome de shock tóxico debido a piomiositis ${ }^{4}$. Al examen físico no se encuentran adenopatías regionales ${ }^{3}$.

Las anormalidades en el laboratorio difícilmente colaboran en establecer el diagnóstico pudiendo presentarse leucocitosis y eritrosedimentación elevada; la eosinofilia suele observarse en la piomiositis tropical pero es infrecuente en la no tropical. Las concentraciones plasmáticas de creatin-fosfoquinasa, aldolasa y aspartato aminotransferasa suelen ser normales a pesar que se produzca una extensa mionecrosis ${ }^{4,13}$, lo cual permite la exclusión de miositis de otras etiologías ${ }^{14}$.

El diagnóstico requiere una alta sospecha y se confirma con imágenes (TAC y resonancia magnética) o bacteriología, tanto con hemocultivos o punción muscular ${ }^{15}$. Los hemocultivos son positivos en 5 a $30 \%$ de los $\operatorname{casos}^{16}$.

Los patógenos aislados con mayor frecuencia son: $S$. aureus, Streptococcus pyogenes y bacilos gramnegativos aeróbicos ${ }^{17}$; otras etiologías son: especies anaerobias, micobacterias (M. avium complex y M. tuberculosis), Microsporidia y hongos (Cryptococcus neoformans, Aspergillus spp., Candida spp., Fusarium spp., y Pneumocystis jiroveci $)^{4}$. Staphylococcus aureus es el patógeno causante en $90 \%$ de los casos $^{7}$, tanto en pacientes inmunocompetentes como inmunocomprometidos ${ }^{1}$.

Se establece el diagnóstico durante la cirugía o mediante aspiración guiada por imágenes ${ }^{7}$.

El tratamiento habitualmente consiste en antimicrobianos y drenaje quirúrgico ${ }^{3,11}$. Si la enfermedad se reconoce en los estadios tempranos, usualmente el tratamiento antiinfeccioso es suficiente, debiendo incluir una cobertura contra $S$. aureus y será modificada subsecuentemente en base a cultivos y susceptibilidad in vitro ${ }^{3}$. Es recomendable utilizar la combinación de dos antimicrobianos efectivos contra $S$. aureus.

La duración del tratamiento no se encuentra estableci$\mathrm{da}^{18}$, generalmente se aplica durante tres a cuatro semanas, con terapéutica ev inicial, pero puede variar considerablemente de acuerdo a la extensión de la enfermedad ${ }^{4}$. El pronóstico es excelente, con la excepción de los casos diagnosticados en estadios avanzados, que cursan con shock y fallo multiorgánico ${ }^{3}$.

La mortalidad varía entre 0,89 y 10\% según diferentes series, siendo la sepsis la principal causa de muerte ${ }^{7}$.

Inicialmente no es posible palpar el absceso dado que se encuentra en la profundidad del músculo, pero el área se encuentra firme, asociada con dolor y rigidez ${ }^{16}$.

En los últimos veinte años los reportes de piomiositis bacteriana en áreas templadas se han incrementado por lo que el reconocimiento temprano, el apropiado tratamiento antimicrobiano y el drenaje del absceso, en caso que sea indicado, reducen la morbilidad y mortalidad ${ }^{2,4}$.

\section{Resumen}

Se presenta un caso de piomiositis en un varón de 59 años, inmunocompetente, producida por Staphylococcus aureus sensible a meticilina, afectando los músculos pectorales del hemitórax derecho. Fue tratado con drenaje quirúrgico y cefalexina. La piomiositis es una infección infrecuente y potencialmente seria que requiere de un reconocimiento temprano y tratamiento oportuno.

\section{Referencias}

1.- Fox L P, Geyer A S, Grossman M E. Pyomyositis. J Am Acad Dermatol 2004; 51 : 308-14.

2.- $\quad$ Wong S L, Anthony E Y, Shetty A K. Pyomyositis due to Streptococcus pneumonia. Am J Emerg Med 2009; 27: 633-5.

3.- Crum-Cianflone N F. Infectious myositis. Best Pract Res Clin Rheumatol 2006; 20: 1083-96.

4.- Crum N F. Bacterial pyomyositis in the United States. Am J Med 2004; 117: 420-8.

5.- Niamane R, O Jalal, El Ghazi M, Hssaida R, Had A. Multifocal pyomyositis in an inmunocompetent patient. Joint Bone Spine 2004; 71: 595-7.

6.- Hall R L, Callghan J J, Moloney E, Martínez S, Harrelson J. Pyomyositis in a temperate climate. Presentation, diagnosis, and treatment. J Bone Joint Surg Am 1990; 72: 1240-4.

7.- Torralba K D, Quismorio F P Jr. Soft tissue infections. Rheum Dis Clin North Am 2009; 35: 45-62.

8.- Harbarth S J, Lew D P. Pyomyositis as a nontropical disease. Curr Clin Trop Dis 1997; 17: 37-50.

9.- Geelhoed G W, Gray H, Alavi I A, Lattoo H. Pyomyositis: tropical and nontropical. N Engl J Med 1971; 284: 853-4.

10.- Harbarth S J, Lew D P. Pyomyositis as a non tropical disease. Curr Clin Trop Infect Dis 1997; 17: 37-50.

11.- Wilson D. Soft tissue and joint infection. Eur Radiol 2004; 14: E64-E71.

12.- Villamil-Cajoto I, Maceriras-Pan F, VillaciánVicedo M J. Piomiositis: presentación de 17 casos en niños y adultos. Rev Med Chile 2006; 134: 31-4.

13.- Dhillon S S, Adachi R. A 62 year old man with shoulder pain. Chest 2004; 126: 614-16.

14.- Jozefkowicz M, Jorrat P, Méndez J. Piomiositis primaria por Sthaphylococus aureus meticilino-resistente proveniente de la comunidad. Arch Argent Pediatr 2008; 106: 533-51.

15.- Swartz M N. Myositis, Pyomyositis. En Mandell, Douglas \& Bennett’s Principles and Practice of Infectious Diseases. Mandell GL, Douglas RG, Dolin R, eds. Fifth ed. Philadelphia: Churchill Livingstone INC; 2000: 1058-60.

16.- Stevens D L, Bisno A L, Chambers H F, Everett E D, Dellinger P, Goldstein E J C, et al. Guidelines for skin and soft-tissue infections. Clin Infect Dis 2005; 41: 1373-406.

17.- Napolitano LM. Severe soft tissue infections. Infect Dis Clin North Am 2009; 23: 571-91.

18.- Canalejo E, González-Bachs E, Picón M, Carratalá C. Tumoración de partes blandas y fiebre en paciente con síndrome mielodisplásico. Enferm Infecc Microbiol Clin 2005; 23: 105-6. 